\title{
Spin-Polarized STM: Imaging Magnetic Nanostructures with Atomic Resolution
}

\author{
M. Bode*
}

* Institute of Applied Physics, University of Hamburg, 20355 Hamburg, Germany

The experimental progress in spin-polarized scanning tunneling microscopy (SP-STM) - a magnetically sensitive imaging technique with ultra-high resolution-allows the investigation of surfaces, thin films, and epitaxial nanostructures with unforeseen precision [1]. Together with its high surface sensitivity, the atomic resolution capability of SP-STM makes it particularly suited for the investigation of domain structures of antiferromagnetic and superparamagnetic surfaces which could only be studied in some rare cases with moderate spatial resolution in the past because of the lack of macroscopic magnetization.

By choosing appropriate substrates and growth conditions could prepare Fe nanostructures with a wide range of magnetic properties including of ferro-, antiferro-, and superparamagnets. For example, we have recently shown by spin-polarized scanning tunneling microscopy that the Fe monolayer on $\mathrm{W}(001)$ is $c(2 \times 2)$ antiferromagnetic, i.e., it exhibits a checkerboard pattern of antiparallel magnetic moments (see Fig. 1). As can be concluded from measurements in an external magnetic field the easy magnetization axis is out-of-plane [2]. On this antiferromagnetic Fe layer we have occasionally observed phase domain walls which are typically very short and clamped between defects like adsorbates or islands. Our results demonstrate that atomic resolution SP-STM can also be applied to non-periodic and non-collinear spin structures. The domain wall width amounts to 6-8 atomic rows only and the walls are centered between two atomic rows (Fig. 2). The results are compared to Monte-Carlo simulations. While walls oriented along (100) directions are found to be fully compensated, the detailed analysis of (110) walls reveals an uncompensated perpendicular magnetic moment. This may be of great technological importance as uncompensated moments on antiferromagnetic surfaces are responsible for the exchange-bias effect which is widely used in state-of-the-art magnetic storage devices.

\section{References}

[1] M. Bode, Rep. Prog. Phys. 66, 523 (2003).

[2] A. Kubetzka et al., Phys. Rev. Lett. 94 (2005) 087204. 

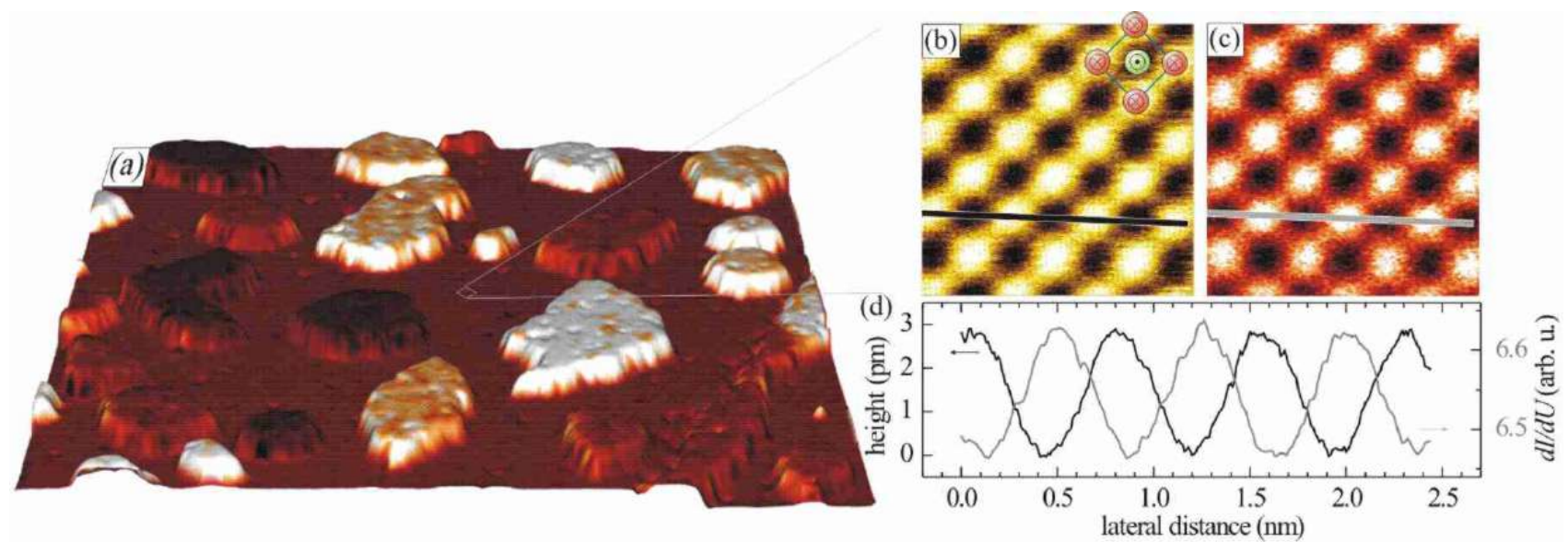

FIG. 1. (a) $3 \mathrm{D}$ composite of topography (height) and $d I / d U$ signal (color) of a $100 \times 100 \mathrm{~nm}^{2}$ surface area of 1.3 ML Fe/W(001). At zero field a four stage magnetic contrast is observed on the second layer islands. In an external field of $B=2.5 \mathrm{~T}$ the $\mathrm{ML}$ shows a $c(2 \times 2)$ superstructure in (b) the constant current image as well as in (c) the corresponding $d I / d U$ map. (d) Line sections along the [100] direction.
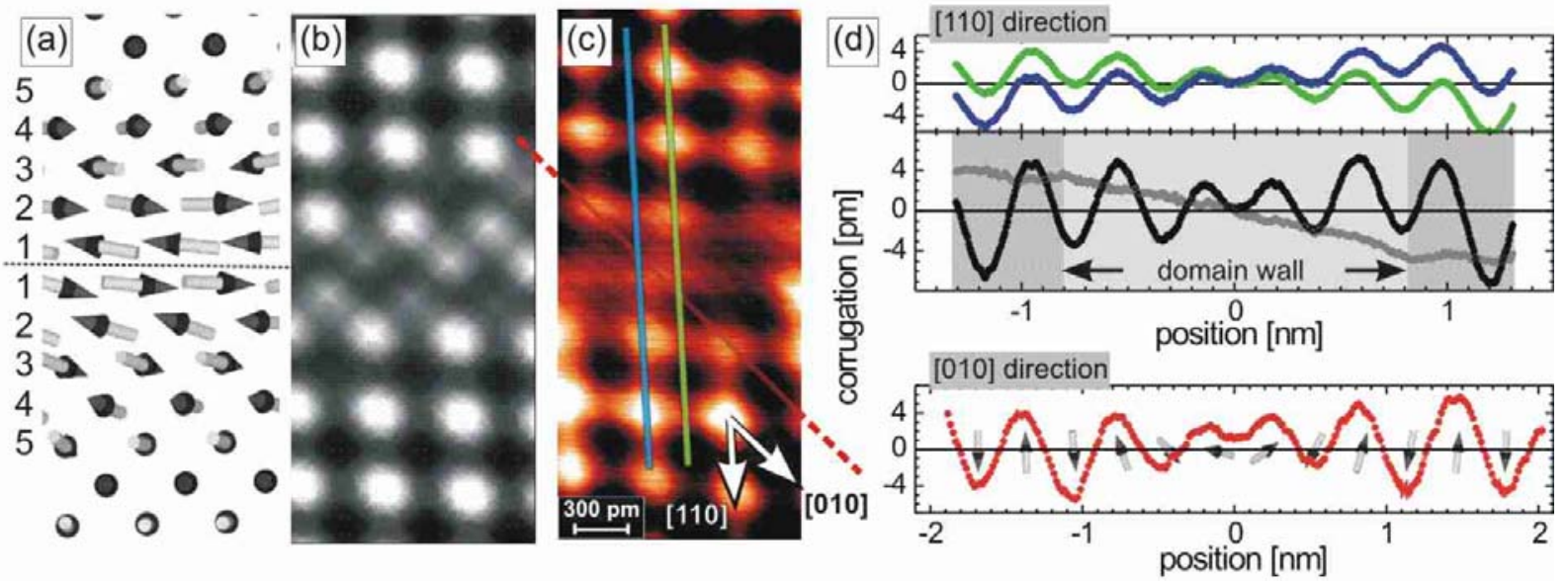

FIG. 2. (a) Theoretical spin structure, (b) simulated, and (c) experimental SP-STM image of a phase domain wall in the out-of-plane antiferromagnetic Fe monolayer on W(001). (d) Height profiles drawn at the positions of the correspondingly colored lines in (c) along the [110] (upper panel) and the [010] direction (lower panel). Middle panel: Sum (black) and difference (gray) of the line profiles shown in the upper panel. The wall is about $1.6 \mathrm{~nm}$ wide and its out-of-plane component exhibits mirror symmetry. 